\title{
Determinantes del acceso a Internet en Colombia*
}

\author{
Determinants of Internet access in Colombia \\ Determinantes do aceso à Internet em Colômbia
}

Recibido el 28 de julio de 2014, aceptado el 10 de octubre, 2014

\section{Héctor Alberto Botello Peñaloza** \\ Colombia}

\section{Resumen}

Objetivo: identificar los determinantes del acceso a Internet en los hogares colombianos durante el 2013. Metodología: se incorporaron las características socioeconómicas dentro de un modelo de elección discreta, con el fin de realizar estimaciones sobre la probabilidad del acceso a Internet en los hogares colombianos. Esto se hizo con base en los microdatos de la encuesta de calidad de vida realizada por el DANE. Resultados: se encontró un aumento notorio del uso de Internet en Colombia: en 2007, el 17\%

Para citar este artículo:

Botello Peñaloza, Héctor Alberto

(2014). Determinantes del acceso a Internet en Colombia. Ánfora, 21(37), 21-36. Universidad Autónoma de Manizales. ISSN 0121-6538 de los colombianos tenía Internet en sus hogares, mientras que en el 2013 llega al 35,66\% de la población. El ingreso es el indicador con mayor efecto en el acceso a Internet. El estudio también evidencia brechas de género significativas, ya que el acceso a este servicio resulta ser mayor para los hombres que para las mujeres. Igualmente, se detectaron amplias diferencias entre localizaciones y niveles educativos

\footnotetext{
* Esta investigación que hace parte del trabajo de la tesis de Maestría terminada sobre el efecto de las tecnologías de la información y la comunicación sobre el desarrollo económico.

** Economista. Magister en Ingeniería, Industrial. Profesor de Política Monetaria y Fiscal. Universidad Industrial de Santander. Perteneciente al grupo de investigación sobre desarrollo regional y ordenamiento territorial y al grupo de investigación en población, ambiente y desarrollo. Correo electrónico: hectoralbertobotello@gmail.com
} 
en relación con el acceso a Internet. Conclusiones: el acceso y tenencia del servicio de Internet ha venido aumentando en Colombia y en América Latina. Sin embargo, dicho proceso no se ha dado de manera homogénea para diferentes grupos de la población. El estudio también concluye que la reducción de las inequidades en relación con el acceso a las TIC, seguirá dependiendo, por mucho tiempo, de soluciones colectivas o públicas.

Palabras clave: Brecha digital; Difusión de Internet; Términos de interacción; América Latina.

\begin{abstract}
Objective: to identify the determinants of Internet access in Colombian homes during 2013. Methodology: socioeconomic characteristics were incorporated within a discrete choice model in order to estimate the probability of Internet access in Colombian households. It was based on microdata from the quality of life survey conducted by DANE (The National Administrative Department of Statistics). Results: a marked increase in the use of Internet in Colombia was found: in 2007, 17\% of Colombians had Internet access at home, and in 2013 the access increased to 35.66\%. The income is the indicator with the highest impact on Internet access. The study also shows significant gender gaps, since men access more to this service then women. Similarly, there were large differences between locations and educational levels in relation to Internet access. Conclusions: internet access and its service ownership have been increasing in Colombia and Latin America. However, this process has not happened uniformly for different population groups. The study also concludes that reducing inequalities in relation to access to ICT will continue, for long, depending on collective or public solutions.
\end{abstract}

Keywords: Digital gap; Dissemination of Internet; Interaction terms; Latin America.

\title{
Resumo
}

Objetivo: identificar os determinantes do aceso à Internet nos lares colombianos durante o 2013. Metodologia: se incorporaram as características socioeconômicas dentro de um modelo de eleição discreta, com o fim de realizar estimações sobre a probabilidade do aceso à Internet nos lares colombianos. Isto se fez baseado nos micro dados da enquete de qualidade de vida realizada pelo DANE. Resultados: encontrou se um aumento notório do uso da Internet em Colombia: em 2007, o $17 \%$ dos colombianos tinha Internet em seus lares, enquanto que em 2013 chega ao $35,66 \%$ da povoação. O ingresso é o indicador com maior efeito no aceso à Internet. O estudo também evidencia brechas de gênero significativas, já que o aceso a este 
serviço resulta ser maior para os homens que para as mulheres. Igualmente, se detectaram amplas diferencias entre localizações e níveis educativos em relação com o aceso à Internet. Conclusões: o aceso e tendência do serviço da Internet têm veio aumentando em Colômbia e em América Latina. Contudo, esse processo não se tem dado de maneira homogênea para diferentes grupos da povoação. O estudo também conclui que a redução das iniqüidades em relação com o aceso às TIC, seguira dependendo, por muito tempo, de soluções coletivas ou públicas.

Palavras clave: Brecha digital; Difusão da Internet; Términos de interação; América Latina. 


\section{Introducción}

El aumento significativo del acceso a Internet ha conlleva cambios en múltiples dimensiones en la sociedad (Silvera, 2005). Por ejemplo, muchas de las actividades que requerían presencia física se han podido simplificar a través de la digitalización. Ahora es posible trabajar, estudiar, pagar cuentas y comprar desde el hogar. Estos fenómenos generan externalidades positivas que pueden aumentar la innovación y la productividad en la economía de un país. Sin embargo, para que esto realmente ocurra, no sólo se debe contar con acceso a Internet sino que además su acceso debe estar asociado a actividades que efectivamente generan externalidades positivas. En ese sentido, la búsqueda de información o la difusión de nuevos conocimientos a través de la web tienen efectos positivos, mientras que la pornografía o el Phishing digital no.

Así, la expansión de la utilización de las Tecnologías de la Información y la Comunicación (TIC) dentro de la población ha venido cobrando fuerza como objeto de estudio para el diseño de políticas para investigadores y académicos, entre otros actores de la sociedad, principalmente referidas al acceso de las TIC, tanto por hogares, como por individuos. Por un lado, se observa que la difusión de las TIC en la población fomenta el empoderamiento de los hogares y territorios, ya que la falta de oportunidades se identifican con altos niveles de pobreza, tal y como lo ha propuesto el premio nobel Amartya Sen. En su pensamiento, el nobel define que las estrategias que apunten a la creación de mayores oportunidades, como la promoción del empoderamiento y el fortalecimiento del capital social, son consideradas políticas públicas básicas para la disminución de las desigualdades sociales y la pobreza (Sen, 2000).

La difusión de las TIC dentro de la población puede contribuir de forma importante al desarrollo de estas estrategias a través de diversos mecanismos (Navarro, 2011). En primer lugar, se reconoce la capacidad de las TIC para ampliar el acceso a diferentes servicios que se pueden brindar de manera digital, tales como las finanzas, el comercio y nuevas oportunidades de trabajo, etc. En segundo lugar, las TIC pueden ampliar el concepto de comunidad, expandiendo las conexiones sociales por fuera de los límites geográficos. Por ejemplo, las familias pueden contactar con sus parientes y amigos en todo el mundo, utilizando teléfonos celulares, correo electrónico, etc. Estas comunidades virtuales han transformado la forma como se crean y mantienen las redes sociales de confianza y de ayuda, conocido como capital social. En tercer lugar, las TIC permiten aumentar la participación en las decisiones de política, al ampliar el acceso a la información, formando mejores actores sociales. Al mismo tiempo, facilita conocer los problemas locales y globales, estrechar relaciones con otros grupos y fortalecer la organización e inserción en acciones colectivas. 
Sin embargo, en las sociedades de América Latina, existe una alta marginación de la población hacia el acceso de los componentes TIC existentes (Silvera, 2005). A este fenómeno se le conoce como brecha digital. La existencia de este fenómeno es considerado una problemática para la mayor parte de los gobiernos pues es un instrumento potencial para exacerbar las desigualdades. Por lo anterior, los gobiernos han justificado su actual en el mercado de las comunicaciones bajo este argumento, impulsando políticas de promoción al acceso (Servon, 2002). A nivel internacional, se ha hecho seguimiento a la existencia de brechas digitales entre países (Ono y Zovodny, 2007; Peres y Hilbert, 2009). Estos autores encuentran que la expansión del acceso en TIC ya están avanzando en los países en desarrollo a una velocidad dos veces mayor que en los desarrollados. Esto se debe a que estos países no tienen necesariamente que repetir las grandes inversiones hechas por los países desarrollados. Pueden evitar la implementación de tecnologías intermedias, como los cables de cobre y los teléfonos analógicos, utilizando directamente tecnologías inalámbricas que requieren menos inversiones y mantenimiento (Filho, 2002).

Igualmente, se han emprendido investigaciones sobre las diferencias en el acceso al interior de grupos dentro de una misma sociedad (Balboni et al., 2011). Por ejemplo, en Latinoamérica, esta problemática ha sido abordada desde una perspectiva del ingreso, por Silvera (2005), Agostini y Willington (2010), por Grazzi y Vergara (2011) y por Grazzi (2011); mientras que autores como Sánchez (2010) exploran las brechas de género en el acceso a las TIC.

Para los primeros, la brecha digital es significativa entre personas con diversos niveles socioeconómicos en los países en vías de desarrollo en general y en Latinoamérica en particular. En relación con el género, la evidencia sugiere que, en promedio, las mujeres sienten menos confianza y menos habilidades frente al acceso a Internet con respecto a los hombres (Hargittai y Shafer, 2006). No obstante, las diferencias de género no solamente dependen de las habilidades de hombres y mujeres para usar las herramientas; hay evidencia de que, incluso, si hay acceso a las TIC en el hogar, las mujeres tendrían en promedio menores oportunidades de acceso a dichas tecnologías (Chen y Wellman, 2004; OECD, 2007). Esto parece estar determinado por los roles de género que deciden el acceso del tiempo de hombres y mujeres. Por ejemplo, las encuestas de acceso del tiempo han podido identificar que las mujeres tienen, en promedio, más horas de trabajo (incluyendo remunerado y no renumerado) y, por ende, menos horas disponibles para el acceso de Internet. Por otro lado, Kennedy et al. (2003) y OECD (2007), encontraron que en Canadá y Estados Unidos, y Grazzi (2011) y Sánchez (2010) en Latinoamérica, que las mujeres utilizan Internet más para fines sociales, de salud y de cuidado de los menores, mientras que los hombres se concentran en actividades de entretenimiento. 
En resumen, se ha expuesto cómo las TIC son elementos que pueden beneficiar el desarrollo de los países al disminuir las inequidades de la sociedad. Sin embargo, no existe literatura sobre los determinantes del acceso del Internet en los hogares colombianos. Por eso, la presente investigación busca estimar los determinantes que motivan la tenencia de Internet en los hogares colombianos entre 2007 y 2013. La importancia de este estudio radica, entonces, en la capacidad de detectar las poblaciones que se ven excluidas en el acceso a las nuevas tecnologías. Esto podría conducir a elaborar políticas que permitan reducir las inequidades sociales, ya que la identificación precisa de estos grupos sociales o regiones geográficas podrían contribuir a la focalización del fenómeno de estudio propagando las ventajas.

Para lograr lo anterior, se propone una aproximación metodológica cuantitativa a través de un modelo de interacciones sobre los microdatos de las encuestas de hogares del DANE (2014) sobre utilización del tiempo libre y de consumo cultural. Este modelo permite la identificación simultánea de los grupos que acceden a las TIC con base en sus características, facilitando el análisis múltiple más allá de los análisis descriptivos.

\section{Metodología}

Primero, se expone la fuente de la que se extrajeron los microdatos y luego se presenta el modelo de estimación implementado, detallando las variables socioeconómicas de los hogares y las interacciones que éstas poseen bajo la hipótesis a evaluar, las cuales fueron extraídas de la literatura reseñada. Posteriormente, con el modelo lineal se prueban estadísticamente dichos aspectos, describiéndolos de manera cuantitativa dentro de las estimaciones.

\section{Fuentes de datos}

El Departamento Administrativo Nacional de Estadísticas (DANE) lleva a cabo la Encuesta de Calidad de Vida (ENCV), las cuales surgen como respuesta a la necesidad de caracterizar la población en los diferentes aspectos involucrados en el bienestar de los hogares. En la ECV 2013 contiene módulos de información de la vivienda, hogares y personas donde se incluyen preguntas asociadas a su actividad económica, indicadores del cuidado en el hogar, seguridad, acceso de las tecnologías de la Información y Comunicación. 


\section{Modelo}

El fenómeno de estudio, el acceso a Internet, se aproxima estadísticamente como una variable categórica, que se enumera como uno en el caso de que la persona haya usado Internet y cero en el caso contrario. Cuando las variables de análisis poseen estas características -la de tener únicamente dos valores- se denominan variables dicotómicas y en su estudio se implementan los modelos de elección discreta.

En general, se considera que detrás de la variable dependiente en estos modelos, $Y$, se encuentra una variable no observable, $I$, que depende de un conjunto de variables explicativas $X_{i}$ que toma ciertos valores si ha sobre pasado cierto lumbral, como se expresa a continuación.

$$
Y_{i}=\left\{\begin{array}{lll}
1 & \text { si } I_{i}^{*}>0 & \text { lo que ocurre cuando } X_{i} \beta+\varepsilon_{i}>0 \\
0 & \text { si } I_{i}^{*}<0 & \text { lo que ocurre cuando } X_{i} \beta+\varepsilon_{i}<0
\end{array}\right.
$$

Donde el supuesto sobre la distribución de $\varepsilon$, determina el tipo de modelo a estimar: si se supone una función de distribución uniforme, se utiliza el modelo lineal de probabilidad truncado; si se distribuye como una normal con media cero y varianza uno, el modelo generado será un probit; mientras que si se supone que es mediante una curva logística, se trataría de un modelo logit. La hipótesis de que el umbral a superar por la variable latente sea cero, se puede modificar por cualquier otro valor sugiriéndose que el valor crítico sea el definido por el término constante (Pérez, 2005). Bajo el primer enfoque el modelo probabilístico quedaría definido (Medina, 2003)

1)

$$
P_{i}=\operatorname{Pr} o b\left(Y_{i}=1\right)=\operatorname{Pr} o b\left(I_{i}^{*}>0\right)=\operatorname{Pr} o b\left(X_{i} \beta+\varepsilon_{i}>0\right)=F\left(X_{i} \beta\right)
$$

La efectividad de estos modelos se mide a través de dos indicadores: el primero es el grado de la varianza de la variable dependiente captada o predicha por parte de las variables independientes, indicador denominado $\mathrm{R}^{2}$. Entre más alto, más efectivo es el modelo en determinar el comportamiento de la variable dependiente y viceversa. El segundo es el grado de observaciones del modelo que han sido clasificadas correctamente en la característica analizada, un mayor porcentaje indica que el grado de robustez del modelo es mejor. En términos estadísticos, hay diferentes situaciones sobre la naturaleza de los datos que se deben corregir para una óptima calibración del modelo tales como la presencia 
de heterocedasticidad, multicolinealidad y autocorrelacion serial (Pérez, 2005). En caso de encontrarse en el conjunto de datos, se procederá a la reestimación corregida de los modelos.

Tomando en cuenta los criterios anteriores, se propone para este trabajo la utilización de la siguiente función probabilística:

$$
\operatorname{Prob}(Y: 1)=f(S, X) \quad=\alpha+\beta \_i X \_i+\varepsilon
$$

Donde $Y$ es la variable a explicar o dependiente categorizada de forma dicotómica. En este modelo toma uno cuando la persona tiene acceso al Internet y cero en caso contrario. Por su parte, $\beta$, captan el aporte marginal a la probidad de acceder al servicio, dado un cambio en cada uno de los factores socioeconómicos que posee el individuo. El factor $\varepsilon$ es el error de la estimación que se distribuye según la función con la que se distribuya de manera logística. El vector de variables independientes resume factores característicos de la persona, $X$, que se seleccionaron con base a la literatura revisada anteriormente:

$\mathrm{H} 1$ : El ingreso es el determinante más importante para que las personas usen Internet, dado que el acceso al mismo se ve limitado en Colombia. Esta variable se representó mediante el ingreso per cápita del individuo.

H2: Existen fuertes diferencias en la demanda de estas tecnologías por departamento y área de localización de la persona. Las diferencias en infraestructura y costes limitan la oferta del servicio a los departamentos con las economías más grandes y en los centros urbanos donde la población está más concentrada.

H3: Diferencias de género. Los hombres tienen una mayor probabilidad de demanda de banda ancha ya que las mujeres suelen concentrarse en las labores del hogar (CEPAL, 2014).

H4: Mayores niveles de educación brindan el conocimiento necesario para aprovechar todos los beneficios de las nuevas tecnologías, además motiva la producción de externalidades positivas asociadas a la globalización del conocimiento y de las actividades que se pueden llevar a cabo de manera digital.

H5: La edad de las personas afecta la demanda de banda ancha, en el sentido en que los niños y jóvenes son más receptivos de estas nuevas tecnologías. En la siguiente sección se proceden a exponer los resultados de las estimaciones, para finalizar con las conclusiones. 


\section{Resultados}

Con base en los ejercicios de estimación, los resultados de este trabajo incluyen las estadísticas sobre el comportamiento del acceso y tenencia de Internet en los hogares colombianos. Finalmente, se presentan los resultados de las estimaciones de los modelos de elección discreta presentados anteriormente.

\section{Estadísticas descriptivas}

El acceso y la tenencia de Internet en el hogar de los colombianos han venido aumentando de manera importante en los últimos años. En la gráfica 1 se aprecia cómo, en 2007, el 17\% de los colombianos tenían Internet en sus hogares mientras que en el 2013 llega al 35,66\%. En relación con el acceso en el 2007,

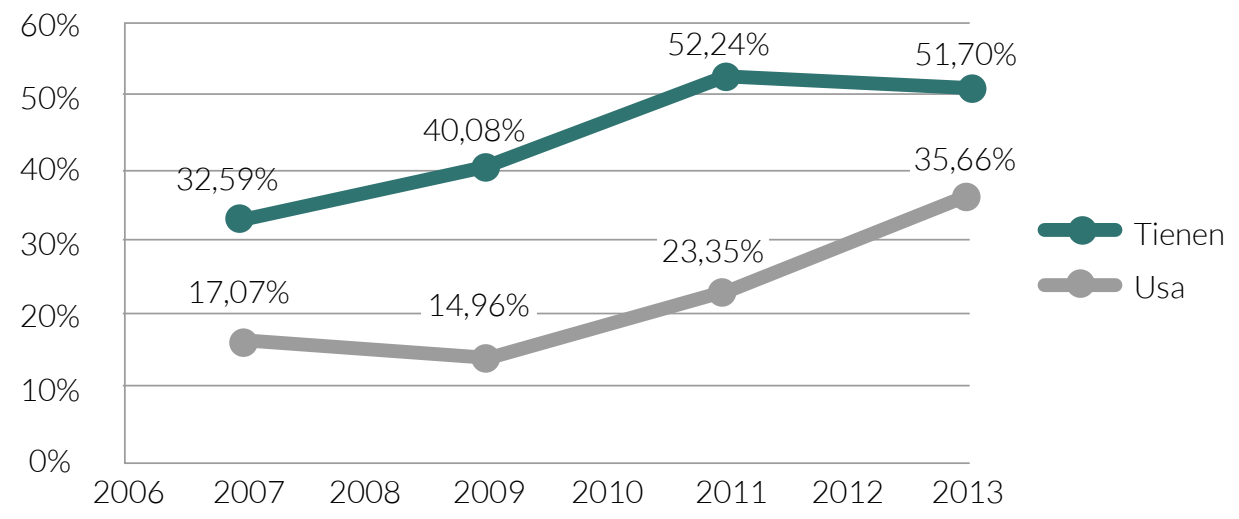

una tercera parte de los colombianos usaba Internet al menos una vez al mes en cualquier lugar (colegios, salas, hogar); en el 2014 este indicador aumentó hasta el $51 \%$ de acuerdo con cifras del DANE (2014).

\section{Gráfica 1. Acceso y tenencia del internet} en los hogares colombianos 2007-2013.

Fuente: elaboración propia con base en los datos de las Encuestas de Calidad de Vida 20072013. Departamento Nacional de Estadística (DANE).

En términos exploratorios, en la tabla 1 se observa el porcentaje de personas que usan Internet al menos una vez al mes por regiones y género, siendo la capital Bogotá la de mayor penetración en el acceso con el $59 \%$ de sus habitantes. Seguidamente se encuentra la región central con el $52 \%$ y la región oriental con el $50 \%$. Esta tendencia permite apreciar las brechas regionales en la demanda de banda ancha que se comentan en la literatura. Igualmente, se observan diferencias entre hombres y mujeres en su posibilidad de acceso a Internet, y en todas las regiones los 
hombres tienen sistemáticamente mayores porcentajes de acceso que las mujeres. Por ejemplo, en Bogotá, el 63\% de los hombres declararon usar Internet una vez al mes, contra un $57 \%$ de las mujeres. Esta diferencia del 6\%, evidencia una brecha de género en el acceso al Internet cercano. El tiempo de acceso a Internet también muestra esta problemática; en la gráfica 2 se puede apreciar que, en promedio, los hombres utilizan el Internet 0,42 horas al día contra un 0,35 horas.

Tabla 1. Porcentaje de personas que usan internet al menos una vez al mes por región y género. Colombia 2013.

\begin{tabular}{|c|c|c|c|}
\hline Región & Hombre & Mujer & Total \\
\hline Bogotá & $63.21 \%$ & $56.97 \%$ & $59.96 \%$ \\
\hline Atlántica & $45.31 \%$ & $43.77 \%$ & $44.53 \%$ \\
\hline Oriental & $53.03 \%$ & $48.86 \%$ & $50.83 \%$ \\
\hline Central & $53.87 \%$ & $48.64 \%$ & $51.13 \%$ \\
\hline Pacífica & $51.11 \%$ & $44.37 \%$ & $47.55 \%$ \\
\hline $\begin{array}{c}\text { Amazonas/ } \\
\text { Orinoquía }\end{array}$ & $50.42 \%$ & $43.70 \%$ & $47.01 \%$ \\
\hline
\end{tabular}

Fuente: elaboración propia con base en los datos de las Encuestas de Calidad de Vida 20072013. Departamento Nacional de Estadística (DANE).

Gráfico 2. Horas diarias promedio de acceso del internet por género y tipo. Colombia 2012.

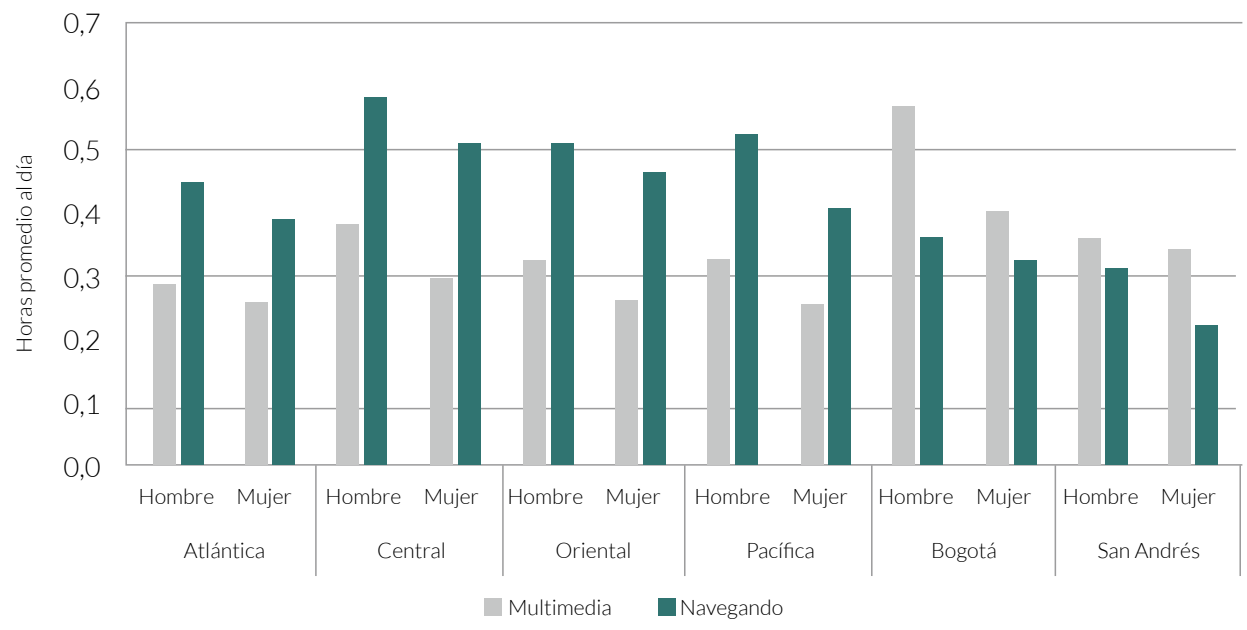

Fuente: Elaboración propia con base en los datos de la Encuesta de acceso del Tiempo (ENUT) 2012. Departamento Nacional de Estadística (DANE). 


\section{Resultado de las estimaciones sobre el modelo econométrico}

Realizando los ejercicios econométricos, se estimaron los determinantes de la demanda de banda ancha en una muestra de $12^{\prime} 872.523$ personas mayores de 12 años. En este orden de ideas, la tabla 2 muestra los resultados de las estimaciones con los coeficientes y los efectos marginales de cada una de las variables de control (independientes) sobre la probabilidad de la persona a acceder al servicio de Internet en 2013. En términos del ajuste global, el modelo resulta aceptable considerando que el valor del estadístico chi2 es muy significativo $\left(3^{\prime} 446,694\right)$. A su vez, todas las variables introducidas presentaron niveles de significancia estadística del 5\% y según el R2 ajustado, el modelo consigue explicar un 52\% de la varianza de la variable dependiente, mientras que el porcentaje de casos correctamente clasificados dentro del modelo propuesto alcanzó cerca del 86\%. Con esta verificación de significancia del modelo, se pasa a comprobar cada una de las hipótesis propuestas por el trabajo contrastándolas con el efecto que tengan las variables independientes con la probabilidad de exportar de la firma.

Para la interpretación de los resultados, el efecto marginal (elasticidad) en las variables continuas equivale al porcentaje en el que se incrementa la probabilidad de acceder al servicio dado un cambio del $1 \%$ en la variable independiente de análisis. Para las categóricas, el efecto marginal mide la probabilidad de manera relativa como comparación de una característica base, por ejemplo, la probabilidad de que una persona ubicada en el área rural use Internet es un 9\% menor que en una cabecera municipal.

Tabla 1. Estimaciones del modelo logit sobre la demanda de internet Colombia 2013.

\begin{tabular}{|c|c|c|c|c|c|c|}
\hline Variable & $\begin{array}{c}\text { Característica } \\
\text { Evaluada }\end{array}$ & Coeficiente & $\begin{array}{l}\text { Efecto } \\
\text { Marginal }\end{array}$ & Error & $\mathrm{P}(\mathrm{z})$ & Característica base \\
\hline Tiene PC & $\mathrm{Si}$ & 1.8317 & 0.2917 & 0.0004 & 0.0000 & No \\
\hline Sexo & Mujer & -0.7264 & -0.0961 & 0.0010 & 0.0000 & Hombre \\
\hline \multirow[t]{7}{*}{ Educación } & Primaria & 0.8684 & 0.1338 & 0.0022 & 0.0000 & \multirow[t]{7}{*}{ Ninguno } \\
\hline & Secundaria & 1.9533 & 0.3837 & 0.0029 & 0.0000 & \\
\hline & Media & 3.0559 & 0.5971 & 0.0024 & 0.0000 & \\
\hline & Técnico & 3.9488 & 0.7526 & 0.0013 & 0.0000 & \\
\hline & Tecnológico & 4.4761 & 0.7856 & 0.0009 & 0.0000 & \\
\hline & Universitaria & 5.2791 & 0.8360 & 0.0006 & 0.0000 & \\
\hline & Posgrado & 6.1501 & 0.8409 & 0.0004 & 0.0000 & \\
\hline \multirow{4}{*}{$\begin{array}{c}\text { Cantidad de } \\
\text { personas en el } \\
\text { hogar }\end{array}$} & 2 & -0.1721 & -0.0236 & 0.0005 & 0.0000 & \multirow[t]{4}{*}{1} \\
\hline & 3 & -0.3160 & -0.0425 & 0.0005 & 0.0000 & \\
\hline & 4 & -0.4674 & -0.0610 & 0.0005 & 0.0000 & \\
\hline & 5 o más & -0.4340 & -0.0554 & 0.0005 & 0.0000 & \\
\hline
\end{tabular}




\begin{tabular}{|c|c|c|c|c|c|c|}
\hline \multirow[t]{2}{*}{ Área } & Centro disperso & -0.1339 & -0.0183 & 0.0012 & 0.0000 & \multirow[t]{2}{*}{ Cabecera } \\
\hline & Rural & -0.7600 & -0.0904 & 0.0009 & 0.0000 & \\
\hline \multirow[t]{3}{*}{ Grupo étnico } & Gitano & -0.5951 & -0.0691 & 0.0045 & 0.0000 & \multirow[t]{3}{*}{ Indígena } \\
\hline & afrodescendiente & -0.1272 & -0.0175 & 0.0010 & 0.0000 & \\
\hline & & 0.1293 & 0.1909 & 0.0010 & 0.0000 & \\
\hline \multicolumn{2}{|c|}{ Horas cuidado familiar } & -0.2949 & -0.0420 & 0.0006 & 0.0000 & Continua \\
\hline \multicolumn{2}{|c|}{ Log (Ingreso per cápita) } & 0.1844 & 0.0262 & 0.0002 & 0.0000 & Continua \\
\hline \multicolumn{2}{|c|}{ Edad } & -0.0806 & -0.0115 & 0.0000 & 0.0000 & Continua \\
\hline \multirow{4}{*}{$\begin{array}{l}\text { Actividad que paso } \\
\text { más el tiempo }\end{array}$} & Buscando & 0.1919 & 0.0290 & 0.0009 & 0.0000 & \multirow[t]{4}{*}{ Trabajando } \\
\hline & Estudiando & 3.5918 & 0.7110 & 0.0039 & 0.0000 & \\
\hline & Oficios & -0.2968 & -0.0398 & 0.0004 & 0.0000 & \\
\hline & Incapacitado & -0.2851 & -0.0371 & 0.0011 & 0.0000 & \\
\hline \multirow[t]{28}{*}{ Departamento } & Atlántico & -0.0980 & -0.0135 & 0.0007 & 0.0000 & \multirow[t]{28}{*}{ Antioquia } \\
\hline & Bogotá, D.C. & 0.3073 & 0.0466 & 0.0005 & 0.0000 & \\
\hline & Bolívar & -0.5101 & -0.0620 & 0.0006 & 0.0000 & \\
\hline & Boyacá & -0.1999 & -0.0267 & 0.0010 & 0.0000 & \\
\hline & Caldas & 0.3424 & 0.0540 & 0.0011 & 0.0000 & \\
\hline & Caquetá & -0.2927 & -0.0378 & 0.0018 & 0.0000 & \\
\hline & Cauca & 0.0752 & 0.0109 & 0.0012 & 0.0000 & \\
\hline & Cesar & -0.2428 & -0.0320 & 0.0010 & 0.0000 & \\
\hline & Córdoba & -0.3662 & -0.0463 & 0.0010 & 0.0000 & \\
\hline & Cundinamarca & -0.0156 & -0.0022 & 0.0008 & 0.0050 & \\
\hline & Chocó & -0.4576 & -0.0559 & 0.0013 & 0.0000 & \\
\hline & Huila & 0.4974 & 0.0821 & 0.0016 & 0.0000 & \\
\hline & La Guajira & -0.4497 & -0.0552 & 0.0009 & 0.0000 & \\
\hline & Magdalena & -0.5538 & -0.0659 & 0.0007 & 0.0000 & \\
\hline & Meta & -0.2592 & -0.0340 & 0.0008 & 0.0000 & \\
\hline & Nariño & 0.0266 & 0.0038 & 0.0011 & 0.0010 & \\
\hline & Norte de Santander & -0.5618 & -0.0669 & 0.0006 & 0.0000 & \\
\hline & Quindío & 0.5959 & 0.1012 & 0.0017 & 0.0000 & \\
\hline & Risaralda & 0.3780 & 0.0603 & 0.0012 & 0.0000 & \\
\hline & Santander & 0.2308 & 0.0351 & 0.0008 & 0.0000 & \\
\hline & Sucre & -0.3107 & -0.0399 & 0.0013 & 0.0000 & \\
\hline & Tolima & 0.3238 & 0.0507 & 0.0011 & 0.0000 & \\
\hline & Valle del Cauca & 0.4114 & 0.0649 & 0.0007 & 0.0000 & \\
\hline & Arauca & 0.2099 & 0.0319 & 0.0018 & 0.0000 & \\
\hline & Casanare & 0.3303 & 0.0521 & 0.0018 & 0.0000 & \\
\hline & Putumayo & -1.0844 & -0.1065 & 0.0013 & 0.0000 & \\
\hline & $\begin{array}{c}\text { Archipiélago de San } \\
\text { Andrés }\end{array}$ & -0.3658 & -0.0460 & 0.0025 & 0.0000 & \\
\hline & Amazonas & -0.2297 & -0.0303 & 0.0018 & 0.0000 & \\
\hline
\end{tabular}




\begin{tabular}{|c|c|l|c|c|}
\hline Observaciones & 12872523 & & Wald chi2(117) & 3446694.20 \\
\hline $\begin{array}{c}\text { Correctamente } \\
\text { clasificadas }\end{array}$ & $86.13 \%$ & & Pseudo R2 & 0.5225 \\
\hline
\end{tabular}

Fuente: Elaboración propia con base en los datos de las Encuestas de Calidad de Vida 20072013. Departamento Nacional de Estadística (DANE).

H1: El ingreso es uno de los determinantes más importantes para que las personas usen Internet, un incremento del $1 \%$ en su ingreso mensual incrementa su probabilidad de usar Internet un $2,62 \%$.

H2: Las diferencias entre departamentos en términos de acceso son evidente. Las personas ubicadas en la región central de país tienen probabilidades sensiblemente más altas, cercanas al $20 \%$ en promedio, de hacer acceso del Internet. Igualmente, los habitantes de los centros urbanos poseen una probabilidad mayor del $10 \%$ de los que se encuentran en el área rural de los municipios.

H3: Tal y como se ha encontrado en la evidencia internacional y en la exploración descriptiva, los hombres muestran mayores probabilidades del acceso a Internet, una mujer cuenta con una diferencia negativa del $10 \%$ en contra. Adicionalmente, se evidencian que mayores horas de cuidado familiar restan a la posibilidad de acceso a Internet, en efecto, si se da un aumento del $1 \%$ en las horas de cuidado familiar (niños o labores hogar) la probabilidad del acceso de internet se reduce un 4,2\%.

H4: Existe una relación positiva entre el acceso a Internet y el nivel educativo. Se observa que las personas que tienen títulos de posgrado tienen un $84 \%$ de posibilidad más, que una persona que no tenga ningún grado educativo, de usar Internet; los universitarios un $83 \%$, secundaria $38 \%$ y primaria un $13 \%$.

H5: La edad tiene una relación negativa con respecto a la probabilidad de usar Internet. Un aumento de un año, disminuye un 1,15\% la posibilidad de usar Internet. Eso quiere decir que las personas más jóvenes son más proclives al acceso a Internet, dada su mayor adaptación a las nuevas tecnologías y el incremento en el acceso de redes sociales y multimedia.

\section{Conclusiones}

La investigación encontró que el acceso y tenencia del servicio de Internet en Colombia ha venido aumentando con el tiempo tal y como es la evidencia encontrada en América Latina (Castillo, 2013). Sin embargo, dicho proceso no se ha dado de manera homogénea para diferentes grupos de la población. Los hogares de mayores ingresos dentro de las áreas urbanas de los departamentos 
de la región central se han vista más favorecidos, gracias a que su ubicación geográfica les facilita el acceso a un mayor stock de infraestructura tecnológica que permite disminuir los costes accediendo de manera más accesible al servicio.

Igualmente, se concluye que el determinante más relevante para el acceso del servicio es el ingreso, resultados que son consistentes con los encontrados para Latinoamérica por Agostini y Willington (2010), Grazzi y Vergara (2011) y Grazzi (2011). Estos hallazgos también soportan la conclusión de que el acceso a internet aún se encuentra reservado para una parte privilegiada de la población que cuenta con el suficiente ingreso para tener servicio en la casa o la utilización de salas, continuando procesos de marginación que continúan en América latina (Silvera, 2005). Además, se evidencia la presencia de la brecha digital de género. La probabilidad de que una mujer use el servicio de Internet es un $9 \%$ más baja que los hombres, siendo esta diferencia similar a la encontrada por Sánchez (2010) en donde es alrededor del 10\%. Para Colombia este resultado se encuentra afectado por el tiempo en que las mujeres utilizan en el trabajo en el cuidado de niños y en los oficios del hogar.

Las limitaciones de la presente investigación giran en torno a la muestra utilizada para ejecutar el modelo, igualmente, la limitación en el número de variables. Las ampliaciones se orientarían a investigar la evolución de los determinantes y las brechas digitales dentro de diferentes grupos. Las variaciones regionales en términos de composición social y cultural pueden afectar las estimaciones agregadas por lo que es un factor a tener en cuenta.

En este orden de ideas, los hallazgos de brechas digitales (tanto geográficamente como por genero) van en línea con la evidencia internacional en países en desarrollo, las cuales apuntan a que la reducción de las inequidades en relación con el acceso a las TIC, seguirá dependiendo, bastante tiempo, de soluciones colectivas o públicas. Un único punto de conexión a Internet puede ser compartido por muchos, dando, por ejemplo, a escuelas que nunca tuvieran una biblioteca un amplio acceso a las mejores bibliotecas del mundo y a sus alumnos la oportunidad de asistir a clases de los mejores profesores. Las políticas públicas en diferentes países latinoamericanos apuntan a la proliferación de servicios de internet localizados en centros comunitarios y culturales, bibliotecas, escuelas, centros de salud y otros espacios públicos, especialmente en zonas marginales y remotas. Algunos centros de conexión ofrecen nuevos paquetes gratuitos de conocimiento en el acceso de TIC, servicios de empleo y desarrollo de aplicaciones.

Estas políticas se basan en la idea de que el acceso a Internet, si bien es privado por cuestión de infraestructura, es un bien público, porque su acceso es una forma de expandir la información, el conocimiento, por lo que debe ser objeto de políticas públicas. Dadas las características de los bienes públicos (no exclusión, no agotamiento), las empresas privadas tienen pocos incentivos para 
invertir en la producción de conocimientos e informaciones, pues no pueden apropiarse totalmente de sus beneficios, a pesar de los intentos cada vez mayores de privatizar el acceso a estos bienes esencialmente públicos. En el momento de decidir dónde invertir, las empresas privadas tienden a privilegiar lo que les reconoce un retorno exclusivo y no lo que les permite a otros beneficiarse de su inversión.

En este orden de ideas, el Estado debe hacer frente en varios aspectos, tales como la creación de la infraestructura básica de comunicaciones, el establecimiento de marcos reguladores, incluidos los estímulos para la participación del sector privado y, especialmente, la provisión de servicios que el mercado difícilmente pondrá en marcha: los de alto beneficio público y alto costo. La educación virtual para la población de más bajos recursos es un buen ejemplo; los costos de montaje de los programas son altos pero los beneficios son de impacto social.

\section{Referencias}

Balboni, M., Rovira, S. y Vergara, S. (Eds.). (2011). ICT in Latin America A Microdata Analysis. Santiago de Chile: ECLAC, United Nations.

Castillo, M. (2013). Estrategias de TIC ante el desafío del cambio estructural en América Latina y el Caribe: balance y retos de renovación.

Comisión Económica para América Latina y el Caribe, CEPAL, (2014). Informe de la quincuagésima Mesa Directiva de la Conferencia Regional sobre la Mujer de América Latina y el Caribe. Santiago, 19 y 20 de mayo de 2014.

Chen W. y Wellman, B. (2004). Th e Global Digital Divide - Within and Between Countries. IT E् Society 1(7), 39-45.

Departamento Administrativo Nacional de Estadística, DANE, (2014). Encuestas de Calidad de Vida 2007-2013.

Filho, A. P. (2002). Inequidades de acceso a la información e inequidades en salud. Rev Panam Salud Publica, 1 1(5/6), 409.

Grazzi, M. (2011). Patterns of Internet use. En M. Balboni, S. Rovira y S. Vergara (Eds.), ICT in Latin America A Microdata Analysis. Santiago: ECLAC, United Nations.

Grazzi, M. y Vergara, S. (2011). Determinants of ICT Access. En M. Balboni, S. Rovira y S.

Vergara (Eds.). ICT in Latin America A Microdata Analysis. Santiago: ECLAC, United Nations. 
Hargittai, E., Shafer, S. (2006). Differences in Actual and Perceived. Online Skills: The Role of Gender. Social Science Quarterly, 87(2), 432-448.

Kennedy, T., Wellman, B., Klement, K. (2003) Gendering the Digital Divide. IT \& Society, 1(5), summer 2003, 72-96.

Medina, E. (2003). Modelos de elección discreta. Publicaciones Económicas de la Universidad Autónoma de Madrid. España, 26.

Navarro, L. (2011). Impact of Internet Use on Individual Earnings. En M. Balboni, S. Rovira y S.

Vergara (eds.). ICT in Latin America A Microdata Analysis. Santiago: ECLAC, United Nations.

Ono, H. y Zovodny, M. (2007). Digital Inequality: A Five Country Comparison Using Microdata. Social Science Research 36(3), 1135-1155.

Peres, W. y Hilbert, M. (2009). La sociedad de la información en América Latina y el Caribe: desarrollo de las tecnologías y tecnologías para el desarrollo. Santiago: ECLAC Books.

Pérez, C. (2005). Métodos estadísticos avanzados con SPSS. Thompson. Madrid.

Sánchez, M. (2010). Implicaciones de Género en la Sociedad de la Información: Un análisis desde los Determinantes de acceso de Internet en Chile y México. Journal of technology management \& innovation, 5(1), 108-126.

Sen, A. (2000). El desarrollo como libertad. Gaceta Ecológica, (55), 14-20.

Servon, L. (2002). Bridging the Digital Divide: Technology, Community, and Public Policy. Malden, MA: Blackwell Publishing.

Silvera, C. (2005). La alfabetización digital: una herramienta para alcanzar el desarrollo y la equidad en los países de América latina y el Caribe. Acimed, 13 (1), 1-1. 